\title{
Motion Control, Planning and Manipulation of Nanowires Under Electric-Fields in Fluid Suspension
}

\author{
Kaiyan Yu, Student Member, IEEE, Jingang Yi, Senior Member, IEEE, and Jerry W. Shan
}

\begin{abstract}
Automated manipulation of nanowires and nanotubes would enable the scalable manufacturing of nanodevices for a variety of applications, including nanoelectronics and biological applications. In this paper, we present an electric-field-based method for motion control, planning, and manipulation of nanowires in liquid suspension with a simple, generic set of electrodes. We first present a dynamic model and a vision-based motion control of the nanowire motion in dilute suspension with a set of $N \times N$ controllable electrodes. Since the motion planning of a nanowire from one position to the target location is NP-hard, two heuristic algorithms are presented to generate near-optimal motion trajectories. We compare the heuristic motion planning algorithms with other existing algorithms such as the rapidly exploring random tree (RRT) and $A^{*}$ algorithms. The comparisons show that the proposed heuristic algorithms obtain near-optimal minimum time trajectories. Finally, we demonstrate a single, integrated process to position, orient, and deposit multiple nanowires onto the substrate. Extensive experimental and numerical results are presented to confirm the motion control and planning algorithms.
\end{abstract}

Note to Practitioners-To fully realize the enormous potential of functional nanodevices, automated, scalable methods are needed to manipulate and assemble nanowires and nanotubes with controlled orientations at specific spatial locations. This paper presents one such technique for the automated motion planning, control and manipulation of individual nanowires suspended in a fluid. The design uses a set of electrode arrays to drive and orientate the nanowire from one location to the target location under electrophoretic and electro-osmotic forces. The motion modeling and control of an individual nanowire in fluid suspension are first presented to guide the nanowire to follow a given trajectory. Then, using the symmetry of the electric-field distribution, we present two heuristic nanowire motion planning algorithms to reduce the computational complexity. The algorithms are demonstrated and validated through experiments. We finally present several experiments demonstrating the steering and positioning of individual and multiple (in sequence) nanowires in a fluid suspension to form geometric patterns. The results will help provide a foundation for scalable, automated methods for manipulating nanowires to build nanodevices.

Manuscript received January 07, 2014; revised April 11, 2014; accepted May 12, 2014. Date of publication June 13, 2014; date of current version December 31, 2014. This paper was recommended for publication by Associate Editor J. Li and Editor L. Shi upon evaluation of the reviewers' comments. The work of J. Shan was supported in part by the National Science Foundation (NSF) Award CBET 0644719. The work of K. Yu was supported in part by a Graduate Fellowship from the Chinese Scholarship Council (CSC). This paper was presented in part at the 2013 IEEE International Conference on Automation Science and Engineering, Madison, WI, USA, August 17-21, 2013, and the 2014 IEEE/ASME International Conference on Advanced Intelligent Mechatronics, Besancon, France, July 8-11, 2014. (Corresponding author: Jingang Yi.)

The authors are with the Department of Mechanical and Aerospace Engineering, Rutgers University, Piscataway, NJ 08854 USA (e-mail: kaiyan.yu@rutgers.edu; jgyi@rutgers.edu; jshan@jove.rutgers.edu).

Color versions of one or more of the figures in this paper are available online at http://ieeexplore.ieee.org.

Digital Object Identifier 10.1109/TASE.2014.2326404
Index Terms-Electro-osmosis (EO), electrophoresis, motion planning, nanowire control, nanowire manipulation.

\section{INTRODUCTION}

$\mathbf{T}$ HE AUTOMATED manipulation and assembly of nanowires or nanotubes is a fundamental challenge for manufacturing functional nanodevices [1]-[3]. Under electric fields, particles in fluid suspension experience an electrophoretic (EP) force proportional to the effective electrokinetic potential (i.e., the zeta-potential) and the field strength, while the fluid itself experiences electro-osmotic (EO) forces [4]. It is important to note that even electrically neutral particles in liquid suspension typically have a nonzero zeta-potential in the interfacial double layer; a large zeta-potential is, in fact, a common stability criterion for colloidal suspensions. Therefore, by actuating a set of electrodes, a precisely controlled electric field can be generated and used to drive nanowires to desired locations under EP and EO forces. The goal of this paper is to present an electric-field-based motion-control strategy for effectively manipulating individual nanowires in dilute suspensions.

Compared with other nanomanipulation and nanoassembly techniques such as the tip-based approach [5], [6], the use of electric-field-induced forces to drive a particle in suspension has advantages of ease and superior scalability. As a result, electrophoresis and dielectrophoresis (DEP) have been explored in the past decade as methods to precisely control nanowire motion in microfluidic devices [7]. Compared to DEP, which depends on the spatial gradient of square of the electric field, EP-based manipulation is simple, requires lower electric fields, and is easier to implement for long-range motion. Most existing work on the use of electric fields to position and sort nanoparticles in fluid suspension have dealt with spherical particles such as beads and biological cells. The electric-field-based manipulation of high-aspect ratio nanowires and nanotubes, although less common, has also been reported in the literature, such as [8]-[10], etc. However, most of the prior works have used the open-loop control of electrodes and do not consider the distributed feedback control of nanowires with programmable electrode arrays.

The application of electric fields to a particle suspension generates EP forces on the particles, as well as EO flow of the fluid itself [4]. In [11], the use of the EO-based flow control to steer and manipulate the particles and nanowires was extensively discussed and demonstrated. The microfluidic device was fabricated to have multiple electrodes to generate EO that steered and oriented particles and nanowires [11] at the center of 
a star-shaped microfluidic channel. EO modeling and optimization algorithms were used to steer and manipulate particles and nanowires in that design. In our design for electric-field-based motion control, both EP force and EO flow are significant to the particle motion. However, due to lack of precise knowledge of the boundary conditions (specifically the zeta-potential on both the particle and device boundaries), exact modeling of both the $\mathrm{EP}$ and EO actuation is difficult in our case. Instead, we design a robust motion-control algorithm that models the EP driving force and simultaneously compensates for the EO-induced motion disturbance.

Motion planning of nanowires with a set of independently addressable electrodes is challenging due to its combinatorial optimization nature [12]. A closely related topic is the routing problem for droplets in digital microfluidic biochips (DMFB) [13], [14]. In DMFB design, the droplets' motion is controlled by turning on and off a set of electrodes for a given roadmap configuration. The motion planning problem in DMFB is to coordinate multiple droplets' motion for minimum time to reach the desired configuration without collision [15]. The motion planning problem considered in this paper is however different from those in the DMFB design in several aspects. First, the motion of nanowires are much more complex than the droplets in DMFB, since the EO- and EP-induced motion is not restricted to only translation in four discrete directions, but occurs in continuous 3D space. Second, complete control of the nanoparticle motion in our case necessitates consideration of the orientation of the nanowire or nanotube, as well as position. Finally, the motion of nanowires under electric fields is controlled not only by the four electrodes within nearest distance but also a set of neighboring electrodes. These differences significantly increase the complexity of EP/EO motion planning and control design. The results of the minimum-time trajectory control of kinematic mobile robots with a finite input set in [16] are built on assumption of individually self-propelled robots. Thus, those results cannot be directly applied to motion planning for EP- and EO-induced nanowire motion with the electric fields generated by multiple electrodes.

In this paper, we first present a dynamic model and motion control of nanowires under EP in an electric-field. We then discuss geometric properties relevant to the electric-field-based single-nanowire motion planning and control problem. Due to the NP-hard complexity, we present two heuristic control solutions and compare with the other well-known motion planning approaches such as rapidly exploring random tree (RRT) and $A^{*}$ algorithms [12]. Extensive experimental and numerical results confirm the performance of the proposed motion planning and manipulation. We finally present a set of experiments to demonstrate the feasibility of using EP-based devices to steer and deposit nanowires to potentially fabricate nanodevices. The main contribution of this work is twofold: First, we present a new design for the motion control, planning and manipulation of nanowires under electric-fields in fluid suspension. The design and algorithms are experimentally and numerically demonstrated, and could serve as enabling techniques for scalable, automated nanowire-based fabrication for many potential applications. Secondly, the formulation and heuristic solutions of the electric-field-based nanowire motion planning under distributed electrode arrays are new and complement the existing results (e.g., routing algorithms for DMFB [13], [14] and minimum-time trajectory control of self-propelled kinematic robots [16]). Compared with the previous conference publications [17], [18], this paper significantly extends the analysis and experimental results.

The rest of this paper is organized as follows. We present the system configuration and the problem formulation in Section II. We then discuss the electric-field modeling in Section III. The motion control of nanowires under EP forces is discussed in Section IV and the motion planning algorithms are presented in Section V. Experimental results are presented in Section VI before we conclude this paper in Section VII.

\section{SyStem CONFIGURATION AND PROBLEM Formulation}

Fig. 1(a) shows the schematic of the microfluidic device. We consider a device with $N \times N$ electrodes on the bottom surface. The array of circular electrodes are fabricated on a glass substrate with equal distance $L$ between centers [Fig. 1(b)]. Each electrode is independently actuated with a DC voltage and for simplicity, the same actuation voltage is applied to all electrodes. The electrode arrays are covered by fluid containing a dilute concentration of nanowires. A glass coverslip is used to cover the fluid and form a flow reservoir.

The use of the electrodes on the nonconducting bottom surface creates an electric field inside the fluid reservoir that is primarily parallel to the substrate surface. Under this largely horizontal electric field, the nanowires orient and move under EP and $\mathrm{EO}$ forces. To deposit nanowires onto the device surface, an additional electrode is fabricated on the top surface, as shown in Fig. 1(a). For a nanowire in fluid suspension at location $\mathcal{S}$ shown in Fig. 1(a), we first actuate the electrode arrays on the bottom surface to steer and drive the nanowire to the targeted horizontal location $\mathcal{T}$. Then, the electrode arrays are turned off and the electrode on the top surface is turned on to align and drive the nanowire vertically until it reaches the bottom surface. Finally, the electrode arrays are turned on again to lay down and deposit the nanowire onto the device surface with the desired orientation.

Fig. 1(c) illustrates the schematic of the electric-field-based nanowire motion control, planning, and manipulation scheme. The nanowire motion control is based on a model of nanowire dynamics under EP-force actuation, with vision-based feedback control of the nanowire positioning. The control system also compensates for the complex 3D EO flow that is unmodeled. The motion planner determines the minimum-time motion trajectory for given target points and nanowire's current position.

We denote the electrode as $e_{i j}$ at the $i$ th row and the $j$ th column, $i, j=1, \ldots, N$. We define the electrode control matrix $\boldsymbol{u}=\left\{u_{i j}\right\}, i, j=1, \ldots, N$, with

$$
u_{i j}= \begin{cases}1, & \text { if } e_{i j} \text { is turned "on" } \\ 0, & \text { if } e_{i j} \text { is turned "off" }\end{cases}
$$

From $\boldsymbol{u}$, the $m\left(m \leq N^{2}\right)$ powered-“on” electrodes are captured by indexing all non-zero elements $u_{i j}$ into $\boldsymbol{U}=\left\{e_{I_{k}}: I_{k}=\right.$ $\left.(i, j), u_{i j}=1\right\}$ with $|\boldsymbol{U}|=m$, where $|\cdot|$ is the cardinality of a set. For nanowire manipulation using the device shown in 


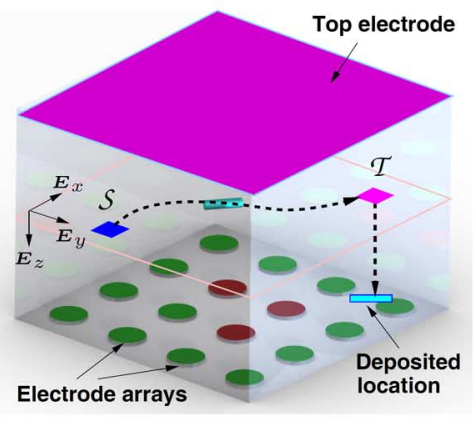

(a)

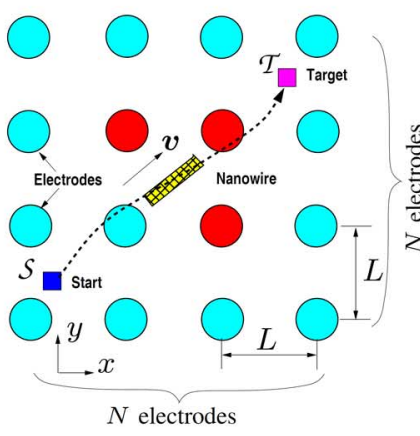

(b)

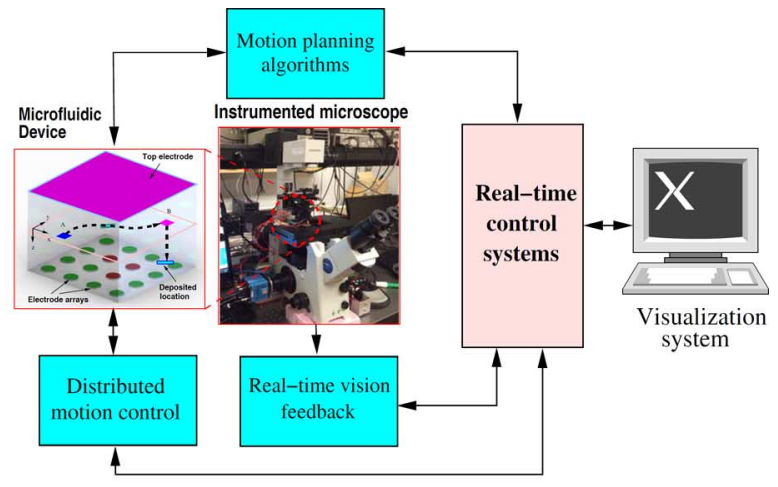

(c)

Fig. 1. Schematic of microfluidic device with $N \times N$ electrodes. (a) 3D view. (b) Top view of the nanowire motion with electrode arrays. (c) A schematic of an electric-field-based nanowire motion control, planning and manipulation scheme.

Fig. 1(a), we mainly consider the following motion control and planning problems.

Motion Control Problem: find electrode control $\boldsymbol{u}(t)$ at time $t$ to steer the nanowire to follow a given trajectory $\mathcal{C}\left(x_{d}, y_{d}\right)$.

Motion Planning Problem: for given starting and target locations $\mathcal{S}$ and $\mathcal{T}$, find a planning path $\mathcal{P}$ that the nanowire takes minimum time from $\mathcal{S}$ to $\mathcal{T}$.

In Sections IV and V, we will present how to solve the above two problems, respectively. In Section III, we first discuss how to efficiently obtain and compute the electric field $\boldsymbol{E}$ at any locations for a given $\boldsymbol{U}$.

\section{ELeCTRIC FIELD MOdeling}

Since Gauss' law for electric fields is linear, the electric-field $\boldsymbol{E}_{p}$ near nanowire $P$ can be calculated by superposition of the electric-field vectors for each power-"on" electrode, namely

$$
\boldsymbol{E}_{p}=\sum_{e_{I_{k}} \in \boldsymbol{U}} \boldsymbol{E}_{k}(P)
$$

where $\boldsymbol{E}_{k}(P)$ is the electric field near $P$ under powered-"on" electrode $e_{I_{k}} \in \boldsymbol{U}$. In the following, we describe how to efficiently compute $\boldsymbol{E}_{p}$.

We consider a nanowire $P$ located at $\boldsymbol{r}_{n}(P)=\left[\begin{array}{ll}x & y\end{array}\right]^{T}$. The position of electrode $e_{i j}$ is denoted as $\boldsymbol{r}_{e_{i j}}$. To determine the electric-field at $\boldsymbol{r}_{n}(P)$, we use the high-fidelity simulation package COMSOL to evaluate the effect of turning on one electrode near $P$. Only activated electrodes within a two-array-distance range $(2 L)$ with respect to nanowire $P$ are found to have a significant effect on the electric field at $\boldsymbol{r}_{n}(P)$, namely, electrodes $e_{i j}$ satisfy $\left\|\boldsymbol{r}_{n}(P)-\boldsymbol{r}_{e_{i j}}\right\|_{1} \leq 2 L$, where $\|\boldsymbol{x}\|_{1}$ is the 1-norm of vector $x \in \mathbb{R}^{2}$. As shown in Fig. 2(a), for nanowire $P$ located inside array cell $A B C D$, the electric field inside $A B C D$ is influenced primarily by electrodes within $A^{\prime} B^{\prime} C^{\prime} D^{\prime}$ shown as the dot-dash lines. For presentation convenience, we define the atomic and basic electrode sets $\mathcal{E}_{a}(P)$ and $\mathcal{E}_{b}(P)$ for nanowire $P$, respectively, as

$$
\mathcal{E}_{a}(P)=\left\{e_{i j}:\left\|\boldsymbol{r}_{n}(P)-\boldsymbol{r}_{e_{i j}}\right\|_{1} \leqslant L\right\}
$$

and

$$
\mathcal{E}_{b}(P)=\left\{e_{i j}:\left\|\boldsymbol{r}_{n}(P)-\boldsymbol{r}_{e_{i j}}\right\|_{1} \leqslant 2 L\right\} .
$$

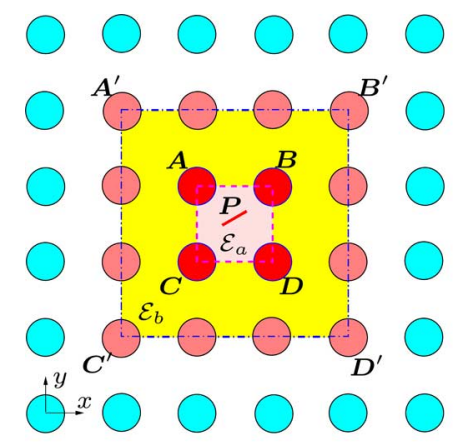

(a)

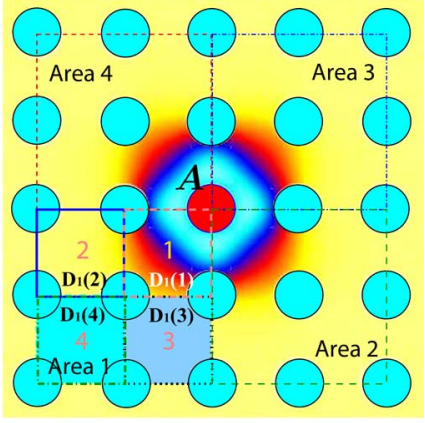

(b)
Fig. 2. (a) A schematic of the atomic and basic areas around nanowire $P$. (b) An illustration of how we compute the electric field by turning on one electrode.

For example, $\mathcal{E}_{a}(P)$ and $\mathcal{E}_{b}(P)$ contain the electrodes in areas $A B C D$ and $A^{\prime} B^{\prime} C^{\prime} D^{\prime}$ in Fig. 2(a), respectively.

To calculate the electric field in $\mathcal{E}_{a}(P)$, we shall compute the electric field generated by turning on each electrode in set $\mathcal{E}_{b}(P) \cap \boldsymbol{U}$ and then superpose them by (2). Notice that the influence of a powered-"on" electrode only affects the electric field within a distance range of $2 L$. Therefore, let us consider the case when one electrode is "on" and find out how to compute the electric field. Fig. 2(b) illustrates a case that electrode $A$ is "on" and all other electrodes in its neighbor within $\mathcal{E}_{b}(A)$ are "off" (i.e., grounded). Due to symmetry, the electric fields are the same around $A$ in four quadrant areas. With this observation, we build the electric field data for one quadrant area (i.e., four atomic cells) and then compute the electric field in the other three quadrant areas by symmetry. We here label region of each atomic electrode cell as $D_{j}(i)$, where index $i=1,2,3,4$ indicates the atomic cell number in each quadrant area and index $j=1,2,3,4$ indicates the left-bottom, right-bottom, right-top, and left-top quadrant areas, respectively, as shown in Fig. 2(b). Moreover, for the four atomic cells $D_{1}(i)$ on the left-bottom corner shown in Fig. 2(b), the electric fields are denoted as $\boldsymbol{E}_{1}(i), i=1,2,3,4$, and assumed to be known.

Algorithm 1 illustrates the computing of the electric field within $\mathcal{E}_{a}(P)$. The algorithm first computes sets $\mathcal{E}_{a}(P)$ and $\mathcal{E}_{b}(P)$. Since the actuated electrodes are given in $\boldsymbol{U}$, the algorithm then finds the subset of powered-"on" electrodes in 
$\mathcal{E}_{b}(P)$, denoted as $\mathcal{E}_{\text {act }}(P)=\mathcal{E}_{b}(P) \cap \boldsymbol{U}$. For each "on" electrode in $\mathcal{E}_{\text {act }}(P)$, the algorithm computes the electric field $\boldsymbol{E}_{i}$ within area $\mathcal{E}_{a}(P)$. By flipping up and down, left and right, or rotating by $180^{\circ}$, we obtain the computation of the electric field $\boldsymbol{E}_{i}(P)$ within $\mathcal{E}_{a}(P)$ for each actuated electrode. Since $\left|\mathcal{E}_{\text {act }}(P)\right| \leq 16$, the complexity of Algorithm 1 is $O(1)$.

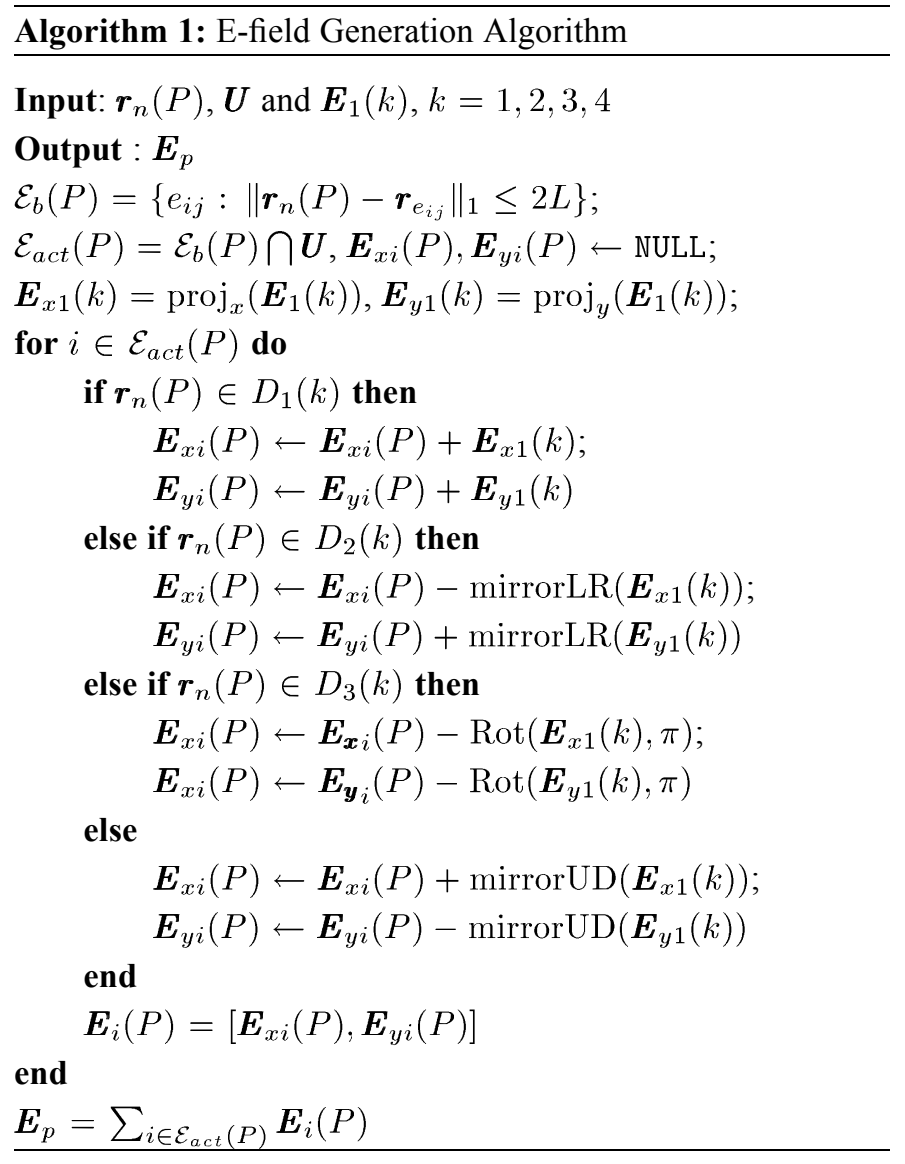

\section{EP-BASEd NANOWire Motion CONTROL}

\section{A. EP-Based Nanowire Motion Model}

We model the nanowire as a prolate spheroid shape, as illustrated in Fig. 3. A body-fixed coordinate system $x y z$ is chosen that is aligned with the principle axes of the ellipsoid. A particle moving in a viscous flow is subjected to hydrodynamic forces. If the movement of the prolate spheroid particle is aligned with its main axis (i.e., $z$ axis), the hydrodynamic drag of the prolate spheroid can be evaluated by [19]

$$
\boldsymbol{F}_{\text {drag }}=\frac{-8 \pi \mu c \boldsymbol{v}}{\left[\left(\frac{a}{c}\right)^{2}+1\right] \operatorname{coth}^{-1}\left(\frac{a}{c}\right)-\frac{a}{c}}=-6 \pi \mu b \boldsymbol{v} R
$$

where $\mu$ is the dynamic viscosity, $\boldsymbol{v}$ is the relative velocity vector of the fluid with respect to the particle, $a$ and $b$ are the major and the minor radii of ellipse, $c=\sqrt{a^{2}-b^{2}}$, and

$$
R=\frac{4}{3 \sqrt{\left(\frac{a}{c}\right)^{2}-1}\left\{\left[\left(\frac{a}{c}\right)^{2}+1\right] \operatorname{coth}^{-1}\left(\frac{a}{c}\right)-\frac{a}{c}\right\}} .
$$

We assume that the nanowire is always aligned and moving tangential to the electric field, as any misalignment from the field

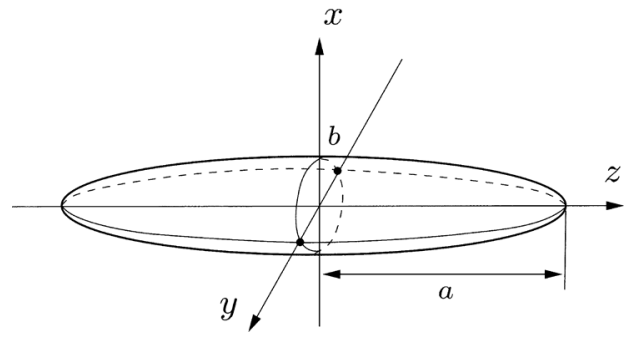

Fig. 3. Prolate spheroid nanowire with major radius $a$ and minor radius $b$.

direction leads to a restoring torque which tends to orient the nanowire with the field [20].

Assuming a thin electric double layer for the particle and no retardation effect, the EP force $\boldsymbol{F}_{E P}$ acting on a particle in liquid suspension is given by

$$
\boldsymbol{F}_{\mathrm{EP}}=6 \pi \mu b \mu_{e} R \boldsymbol{E}=6 \pi b \epsilon_{m} \zeta R \boldsymbol{E}
$$

where $\boldsymbol{E}$ is the DC electric field vector, $\zeta$ is the zeta-potential of the suspended particle (which can be experimentally determined), and $\mu_{e}=\epsilon_{m} \zeta / \mu$, is the electrophoretic mobility, where $\epsilon_{m}$ is the electric permittivity. Due to small Reynolds numbers for the particle motion, the particle is always at steady state and the EP force is balanced by the hydrodynamic viscous drag force, so that $\boldsymbol{F}_{\mathrm{EP}}+\boldsymbol{F}_{\mathrm{drag}}=0$. Using (5) and (6), we obtain

$$
\boldsymbol{v}=\left[\begin{array}{l}
v_{x} \\
v_{y}
\end{array}\right]=\frac{\epsilon_{m} \zeta \boldsymbol{E}}{\mu} .
$$

Brownian translation and rotation of the nanowires are neglected in the model for sufficiently large particle velocities and electric-fields [21]. The motion equation (7) implies that the velocity of the nanowire is independent of the nanowire geometry (e.g., size and shape), provided we can assume a thin double layer and uniform zeta potential [22].

From (7), the velocity magnitude of the nanowire in the suspended plane is proportional to the magnitude of the electric field $\|\boldsymbol{E}\|$, and the direction of motion of the nanowire is along the electric field. By appropriately switching the powered-"on" electrodes, the electric field $\boldsymbol{E}$ can be changed and used to control a nanowire's motion.

\section{B. Nanowire Motion Control}

The goal of the motion control is to steer the nanowire to follow a given trajectory $\mathcal{C}\left(x_{d}, y_{d}\right)$. As shown in Section VI, the nanowire motion is affected significantly by EO flow of the suspending fluid and thus, the control design must be also robust enough to compensate for any unmodeled disturbances.

We extend the potential function approach in [23], [24] to design the path-following control of $\mathcal{C}\left(x_{d}(\tau), y_{d}(\tau)\right)$, which is parameterized by time variable $\tau$, instead of time $t$. We here present the discrete-time motion controller design. The following potential function is used to capture the path-following position errors along $\mathcal{C}$ at the $k$ th step:

$$
U(\boldsymbol{\theta}(k))=\frac{1}{2} \beta_{1}\left[\left(1-\cos 2 \theta_{x}(k)\right)+\left(1-\cos 2 \theta_{y}(k)\right)\right]
$$


where $\beta_{1}>0$ is a constant gain, $\boldsymbol{\theta}(k)=\left[\theta_{x}(k) \theta_{y}(k)\right]^{T}$, $\theta_{x}(k)=\tan ^{-1} e_{x}(k), \theta_{y}(k)=\tan ^{-1} e_{y}(k)$, and the position errors $e_{x}$ and $e_{y}$ are defined as

$$
\boldsymbol{e}(k)=\left[\begin{array}{c}
e_{x}(k) \\
e_{y}(k)
\end{array}\right]=\left[\begin{array}{c}
x(k)-x_{d}(k) \\
y(k)-y_{d}(k)
\end{array}\right] .
$$

The position variables at the $k$ th step for the nanowire and its desired locations are, respectively, as $x(k)=x(k \Delta t), y(k)=$ $y(k \Delta t)$, and $x_{d}(k)=x_{d}\left(\tau_{k}\right), y_{d}(k)=y_{d}\left(\tau_{k}\right)$, where $\Delta t$ is the constant sampling period. The updating law for $\tau_{k}$ will be given later in this section.

At the $k$ th step, the desired velocity $\boldsymbol{v}^{d}(k)=\left[v_{x}^{d}(k) v_{y}^{d}(k)\right]^{T}$ is calculated by

$$
\left[\begin{array}{c}
v_{x}^{d}(k) \\
v_{y}^{d}(k)
\end{array}\right]=\lambda_{1}\left[\begin{array}{c}
\frac{d x_{d}}{d \tau} \\
\frac{d y_{d}}{d \tau}
\end{array}\right]-\lambda_{2}\left[\begin{array}{c}
\sin 2 \theta_{x}(k) \\
\sin 2 \theta_{y}(k)
\end{array}\right]-\lambda_{3}\left[\begin{array}{c}
\hat{e}_{x}(k) \\
\hat{e}_{y}(k)
\end{array}\right]
$$

where $\lambda_{1}=e^{-\beta_{2} U(\boldsymbol{\theta}(k))}, \lambda_{2}=2-e^{-\beta_{2} U(\boldsymbol{\theta}(k))}, \beta_{2}>0$ is a self-pacing parameter and $\lambda_{3}$ is a positive constant parameter. The error prediction, namely, the last term in (10), is calculated by

$$
\hat{\boldsymbol{e}}(k)=\left[\begin{array}{c}
\hat{e}_{x}(k) \\
\hat{e}_{y}(k)
\end{array}\right]=\alpha_{1} \boldsymbol{e}(k)+\alpha_{2}[\boldsymbol{e}(k)-\boldsymbol{e}(k-1)]
$$

where positive constants $\alpha_{1}, \alpha_{2}>0$ are design parameters.

Note that the first term in the right hand of (10) indicates the direction of the desired trajectory $\mathcal{C}$, the second term penalizes the tracking direction and the third term tries to compensate for the predicted error for the next time period using the past twostep error measurements as given in (11). The time parameter updating is given by the following dynamics:

$$
\tau_{k+1}=\tau_{k}+\lambda_{1} \Delta t .
$$

Finally, we need to determine the powered-"on" electrodes such that the electric field $\boldsymbol{E}_{p}(k)$ is to steer nanowire velocity $\boldsymbol{v}(k)$ in (7) to follow $\boldsymbol{v}^{d}(k)$ given by (10). To determine the controlled electrodes, we formulate an optimization approach to determine the actuation electrodes control $\boldsymbol{u}^{*}(k)$ as follows:

$$
\boldsymbol{u}^{*}(k)=\arg _{e_{i j} \in \mathcal{E}_{b}(P)} \min \left\|\boldsymbol{v}_{c}(k)-\boldsymbol{v}^{d}(k)\right\|
$$

where $\boldsymbol{v}_{c}(k)=\epsilon_{m} \zeta \boldsymbol{E}(\boldsymbol{u}(k)) / \mu$ for a given electrode control $\boldsymbol{u}(k)$ for $e_{i j} \in \mathcal{E}_{b}(P)$.

With the above design, we have the following property.

Lemma 1: If the nanowire velocity $\boldsymbol{v}$ follows $\boldsymbol{v}^{d}$ precisely, then path-following errors converge to zero, namely, $\boldsymbol{e}(t) \rightarrow \mathbf{0}$.

Proof: See Appendix A.

Remark 1: The number of elements in $\mathcal{E}_{b}(P)$ is 16 and the most influential electrodes for electric field $\boldsymbol{E}_{p}$ are those in $\mathcal{E}_{a}(P)$, which contains only four electrodes. Therefore, obtaining control $\boldsymbol{u}^{*}$ by searching solution in (13) is not computationally expensive. It is also noted that the powered-"on" electrodes (i.e., $\boldsymbol{u}^{*}$ ) can switch between any consecutive steps. The directions of desired velocity $\boldsymbol{v}^{d}(k)$ and EP-induced velocity $\boldsymbol{v}_{c}(k)$ might not align each other perfectly. Therefore, as shown in experiments in Section VI, the nanowire motion trajectories show zigzag patterns.

\section{EP-BASEd NANowire Motion Planning}

\section{A. Optimal Trajectory Property}

As discussed in Section III, $\boldsymbol{E}_{p}$ is only determined by the 16 electrodes in $\mathcal{E}_{b}(P)$. The motion-planning problem thus becomes to determine "on" or "off" status of these 16 electrodes. However, searching all of the $2^{16}$ possibilities of binary control of electrodes in $\mathcal{E}_{b}(P)$ is prohibitive due to high computational cost. Therefore, we shall use the properties of the motion trajectory to simplify the searching process. It is noted that when an electrode is turned "on," the generated electric field is symmetric about all of the horizontal, vertical and diagonal directions around the electrode. Such symmetry property is used to simplify the planning complexity.

We first introduce some geometry concepts to facilitate the illustration of the motion planning algorithms. Fig. 4 shows the schematic of the motion planning of a nanowire from starting point $\mathcal{S}$ and to target point $\mathcal{T}$. Let us consider the atomic electrode set $\mathcal{E}_{a}=\{S, Q, R, G\}$ in the figure.

Definition 1: A mirror of $\mathcal{E}_{a}$ is a line segment connecting any pair of vertices of $\mathcal{E}_{a}$ or any of the middle points of each side of $\mathcal{E}_{a}$ with its center. A mirror line is the straight-line combined by co-linear mirrors.

For example, for the atomic cell $\mathcal{E}_{a}=\{S, Q, R, G\}$, lines $S W, W G, G V, V R, R U, U Q, Q T, T S, S O, O R, Q O, O G$, $O W, O V, O U$, and $O T$ are all mirrors of $\mathcal{E}_{a}$; mirrors $Q O$ and $O G$ are on the same mirror line $Q G$.

We define the control line as the straight line connecting the nanowire current location and target point $\mathcal{T}$ and the cut line as the line perpendicular to the control line and passing the nanowire current position. For example, lines $\mathcal{S} \mathcal{T}$ and $n n^{\prime}$ are the control line and the cut line, respectively, for the starting and target points $\mathcal{S}$ and $\mathcal{T}$, as shown in Fig. 4. Line $n n^{\prime}$ divides all electrodes into two groups. The electrodes in the half plane $H I J K L$, as shown the shaded area in the figure, are the power sources that drive the nanowire toward $\mathcal{T}$ and we define them as target power electrodes and denote as $\mathcal{E}_{T}$.

Due to symmetry of the electric field in $\mathcal{E}_{a}$, it is straightforward to obtain the following property.

Property 1: For any given trajectory $\mathcal{P}$ in $\mathcal{E}_{a}$, we can always find an alternative electrode control such that a dual trajectory $\mathcal{P}^{\prime}$ achieves the same travel time as that of $\mathcal{P}$, where $\mathcal{P}^{\prime}$ is symmetric with $\mathcal{P}$ about mirror lines of $\mathcal{E}_{a}$.

With the above observation, the minimum time trajectory from $\mathcal{S}$ to $\mathcal{T}$ satisfies the following property.

Lemma 2: The minimum-time trajectory can avoid passing across one mirror line twice.

Proof: See Appendix B.

We now define a trajectory search area $\mathcal{A}_{m}$ as follows.

Definition 2: Trajectory search area $\mathcal{A}_{m}$ is the convex hull whose boundaries are formed by the mirror lines. Moreover, $\mathcal{A}_{m}$ contains all the mirrors that intersect with the control line. If the control line passes through intersection points of mirrors, then take all the intersected mirrors on both sides of the control line into $\mathcal{A}_{m}$; if the control line coincides with any mirrors, then only mirrors lie in either half side of the control line are considered into $\mathcal{A}_{m}$. 


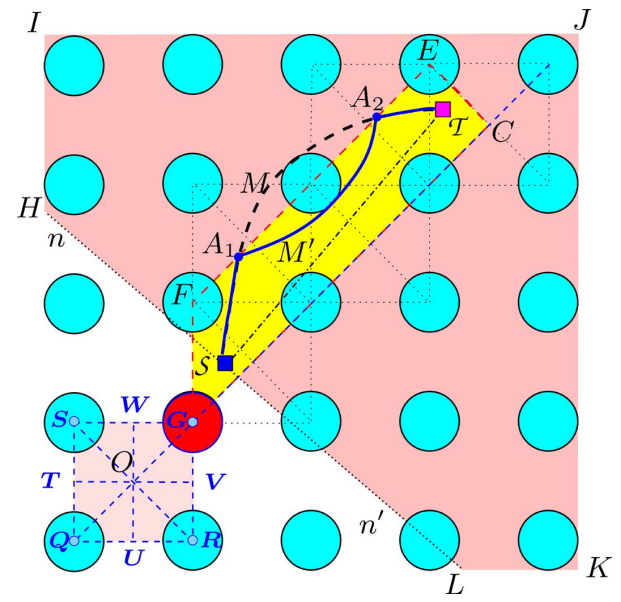

Fig. 4. Illustrative schematic of the trajectory search area.

Using the results in Lemma 2, we have the following property about the minimum-time trajectory.

Lemma 3: The minimum-time trajectory from $\mathcal{S}$ to $\mathcal{T}$ is located inside $\mathcal{A}_{m}$.

Proof: See Appendix C.

For a given pair of starting and target points $\mathcal{S}$ and $\mathcal{T}$, Algorithm 2 describes the computational approach to find $\mathcal{A}_{m}$. The algorithm takes the intersection of the diagonal parallelogram $\mathcal{A}_{d}$, anti-diagonal parallelogram $\mathcal{A}_{a}$, and rectangle $\mathcal{A}_{r}$ areas. All of these parallelograms or rectangles are computed through finding the convex Polygon for sets of lines $\boldsymbol{L}_{h}$ (horizontal with slope $k=0$ ), $\boldsymbol{L}_{v}$ (vertical with slope $k=\infty$ ), $\boldsymbol{L}_{d}$ (diagonal with slope $k=1$ ) and $\boldsymbol{L}_{a}$ (anti-diagonal with slope $k=-1$ ). Function Lineswipe identifies these line sets, and procedure Nearline finds the $i$ th closest lines $\mathbb{L}_{S}$ and $\mathbb{L}_{T}$ to points $\mathcal{S}$ and $\mathcal{T}$ and, with slope $k$ and passing through any electrodes. Procedure Linecross then determines whether these $\mathbb{L}_{S}$ and $\mathbb{L}_{T}$ intersect with control line $\mathbb{L}_{c}$. Function Lineswipe identifies the desired line sets by only testing at most six times for $\mathcal{S}$ and $\mathcal{T}$. The intersection of three polygons costs $O(1)$ since the polygons are either parallelograms or rectangles. Thus, the complexity of the algorithm is $O(1)$.

Algorithm 2: Find trajectory search area $\mathcal{A}_{m}$

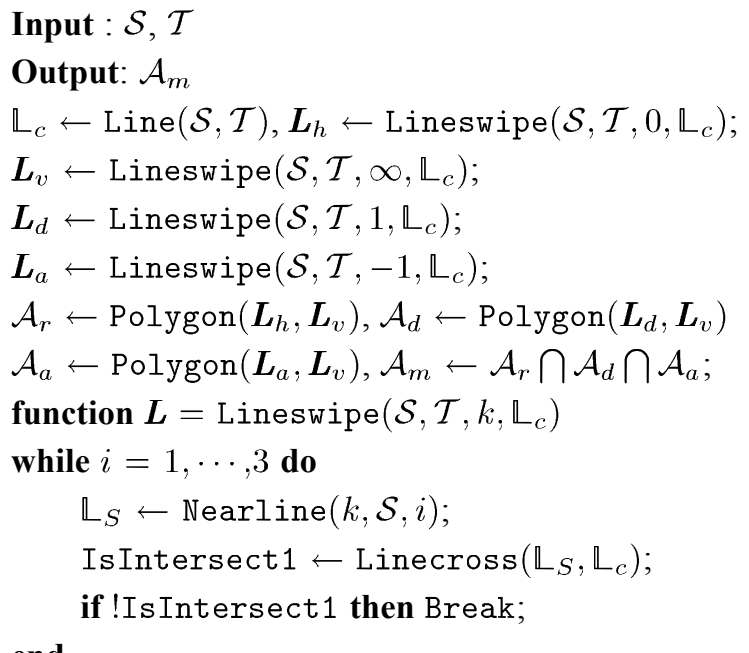

end

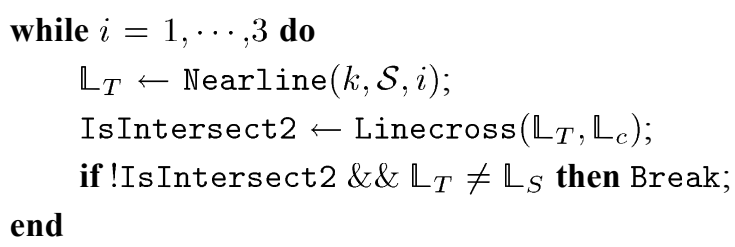

\section{B. Motion-Planning Algorithms}

We here present two heuristic planning algorithms by using the properties described in the previous section. For comparison purposes, we also present the planning results by using the wellknown $A^{*}$ and RRT algorithms.

1) Heuristic Algorithm-AllPower: A heuristic algorithm, AllPower, is simply to turn on all the target power electrodes. At each planning step, the algorithm updates the control line and cut line and then, the target power electrodes are all activated. The nanowire is driven by the maximum velocity along the direction towards $\mathcal{T}$. Although each target power electrode produces an attractive force towards $\mathcal{T}$, the resulting trajectory is not necessarily the shortest time since the travel distance could end up longer than the minimum-time trajectory.

2) Heuristic Algorithm-MinCost: Another heuristic algorithm, MinCost, considers the cost of every possible combination of power sources. At each planning time step, the algorithm computes the velocity field $\boldsymbol{v}_{T}$ and $\boldsymbol{v}_{\perp}$ along the control-line and cut-line directions, respectively, under any possible combination of target power electrodes. The control $\boldsymbol{u}_{c}$ is obtained by maximizing a cost function that penalizes the ratio of $\left|\boldsymbol{v}_{T}\right|$ and $\left|\boldsymbol{v}_{\perp}\right|+v_{0}$, where $v_{0}>0$ is a small constant to avoid singularity, namely

$$
\boldsymbol{u}_{c}=\arg \max _{e_{i j} \in \mathcal{E}_{T}} \frac{\left|\boldsymbol{v}_{T}\right|}{\left|\boldsymbol{v}_{\perp}\right|+v_{0}} .
$$

Under $\boldsymbol{u}_{c}$, a large velocity can be obtained along the control-line direction, while a small velocity in the cut-line direction.

3) $A^{*}$ Pruning: We here modify $A^{*}$ algorithm [12] to solve the planning trajectory. In each planning step, the algorithm expands the $A^{*}$ graph only in $\mathcal{A}_{m}$. For each expended node, it checks whether the node's parents have passed the mirror lines before: if so, deletes the new node that across the same mirror line. Meanwhile, if the new node's position has been explored previously, then the algorithm compares the cost of each trajectory that arrives at that same position, prunes the graph by deleting the higher cost node and reconnects the deleted node's children to the lower cost node. Finally, the algorithm sorts the cost of each node in the open list of the $A^{*}$ graph and drives the nanowire using the least cost. For nanowire $P(x, y)$, the (time) cost function is defined as

$$
T_{c}(P)=\frac{\operatorname{dist}(\mathcal{T}(x, y), P(x, y))}{v_{\max }}+T(P(x, y))
$$

where $\operatorname{dist}(\cdot, \cdot)$ defines the distance between two points, $v_{\max }$ is the possible maximum velocity of the nanowire, $T(P(x, y))$ is the travel time to reach point $P(x, y)$, and $\mathcal{T}(x, y)$ is the target point. It is straightforward to obtain that the cost function never overestimates the minimum time. 


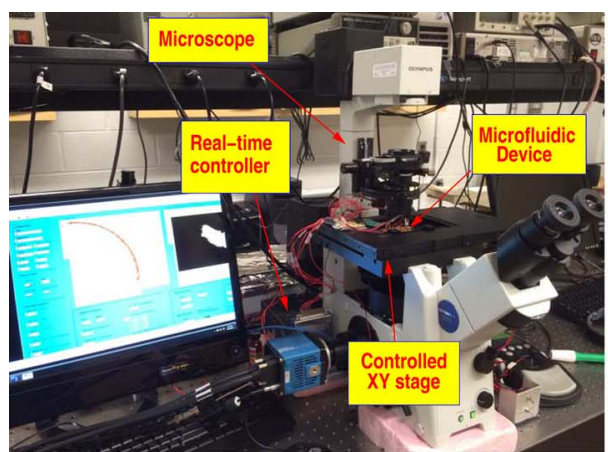

(a)

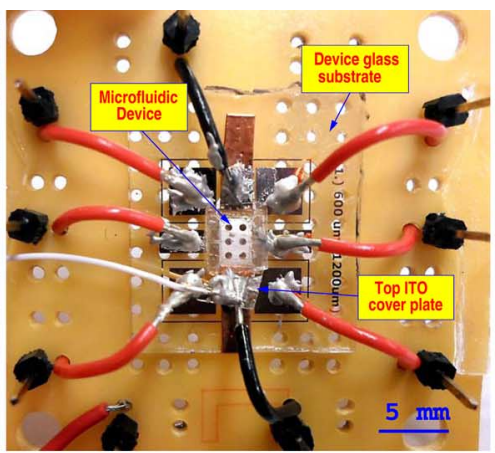

(b)

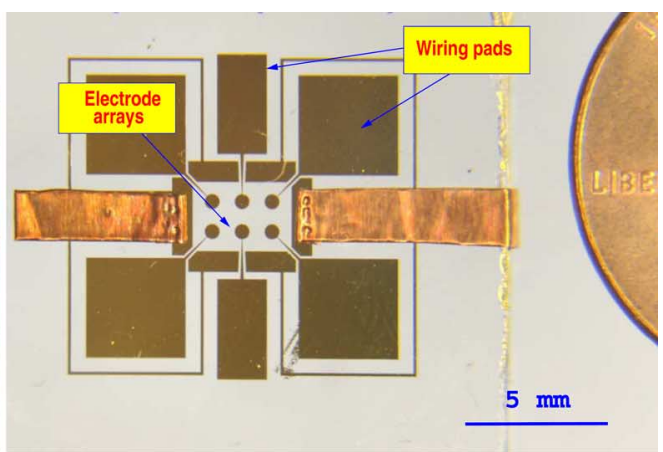

(c)

Fig. 5. The experimental setup. (a) The inverted microscope with embedded control systems and vision-based nanowire motion feedback. (b) The glass microfluidic device is mounted on a PCB for testing and a top coverslip coated with an indium tin oxide (ITO) layer is placed to cover the device. (c) The top view of the microfluidic device with $2 \times 3$ electrode arrays.

4) Breadth-First Search (BFS)-Based Planning: With a fixed planning time step, we build trajectory paths under all possible combinations of powered-"on" electrodes and then a graph is constructed using all possible trajectory paths. A breadth-first search (BFS) method is used to build a searching tree to obtain the optimal results. Similar to the $A^{*}$ algorithm, only the trajectories within $\mathcal{A}_{m}$ are used in the searching process, and mirrorline checking and cost-pruning are also used in this algorithm. At a fixed planning time step, BFS yields the minimum-time result when all the time costs of expanding nodes are equal because it always expands the shallowest unexpanded node. Therefore, we will use BFS as the benchmark to compare various planning algorithms.

5) RRT Pruning: By modifying the sampling based search algorithm [12], the RRT-pruning algorithm only samples points inside $\mathcal{A}_{m}$, enforces the mirror-line checking and employs the cost-pruning technique as those in the $A^{*}$ pruning algorithm to steer the nanowire from the current position to the new nodes and then connect them back to the RRT trees.

\section{EXPERIMENTAL RESULTS}

\section{A. Experimental Setup}

Fig. 5(a) shows the experimental setup for nanowire motion control and manipulation test. A microfluidic device [Fig. 5(c)] with $2 \times 3$ electrode arrays was fabricated on glass and mounted on a PCB connector, as shown in Fig. 5(b). The distance between centers of adjacent electrodes is $L=1200 \mu \mathrm{m}$ and the diameter of each circular electrode is $L / 2=600 \mu \mathrm{m}$. A top coverslip coated with an indium tin oxide (ITO) layer is used to cover the microfluidic device and as an additional electrode to control the vertical position of the nanowires and deposit them onto the bottom surface of the device. An inverted optical microscope (Olympus IX71) with a monochrome CCD camera (PCO AG pco.edge $2560 \times 2160$ pixels) is used to capture and feedback the nanowire trajectory. The PCB connector board is mounted on a motorized, computer-controlled $\mathrm{XY}$ stage (Prior ProScan III). An embedded system (NI cRIO 9074) is used to control the electrode array and a LabView application is developed to process the vision feedback of nanowire positions. Using a voltage source and an amplifier, a $\pm 100 \mathrm{~V}$ DC potential is selectively applied to the electrode array.

We use metal-assisted chemical etched silicon nanowires [25] suspended in heavy viscosity mineral oil at a concentration of $0.5 \mathrm{mg} / \mathrm{ml}$. Fig. 6(a) shows a forest of the silicon nanowires fabricated for the experiments and Fig. 6(b) shows an image of an individual nanowire suspended in the oil, with the extracted contour information by vision algorithms for positioning feedback. The values of the model parameters of the nanowire used in motion control design are as follows: $\mu=251.2 \mathrm{cP}, \epsilon_{m}=2.17 \epsilon_{0}$, $a=2$ to $7 \mu \mathrm{m}, b=100 \mathrm{~nm}$, and sampling time $\Delta t=0.5 \mathrm{~s}$. We use the known electric fields and nanowire motion trajectories to infer the values of particle zeta-potential $\zeta$ in (7). Fig. 7 plots the motion-based estimates of the $\zeta$ values for each of 35 silicon nanowires. The shaded area of Fig. 7 shows the range of zeta-potentials for identical silicon nanowires in oil measured independently using a Brookhaven ZetaPALS instrument. This consistency of the calculated and measured zeta potentials indirectly confirms the basic model of the nanowire dynamics and electric fields described in Sections II and III.

\section{B. Nanowire Motion-Control Results}

Fig. 8 shows the motion control and path-following performance for various trajectories. In experiments, we estimated and updated the zeta-potential values for the nanowire using the motion information of the beginning few seconds. To demonstrate the EO effects in experiments,Fig. 8(a) shows the tracking performance of a straight-line trajectory without disturbance compensation. The motion control of the nanowire shown here is purely based on the EP-model given by (7). More EO flow disturbed motion results are presented in [17]. Clearly, the EO flow disturbance affects the nanowire motion significantly.

Under the motion control (10), Fig. 8(b)-(d) shows the path-following performance of a straight-line, a circular and an "R"-shape trajectory, respectively. Fig. 9 shows the positioning errors for these three trajectories. The errors are consistently within $4 \mu \mathrm{m}$ over a few hundred micrometers range driving distance. For the straight-line following [Figs. 8(b) and 9(a)], the tracking errors are within $1 \mu \mathrm{m}$ most of the time. The plots in these two figures confirm that the motion control design can 


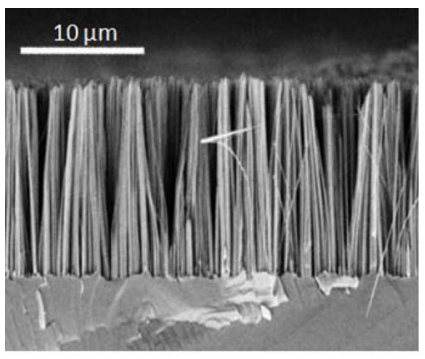

(a)

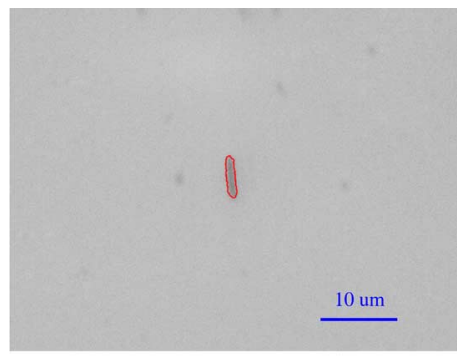

(b)
Fig. 6. (a) SEM image of as-grown silicon nanowires used in experiments. (b) The microscope image of the suspended nanowire, along with the processed contour for positioning feedback.

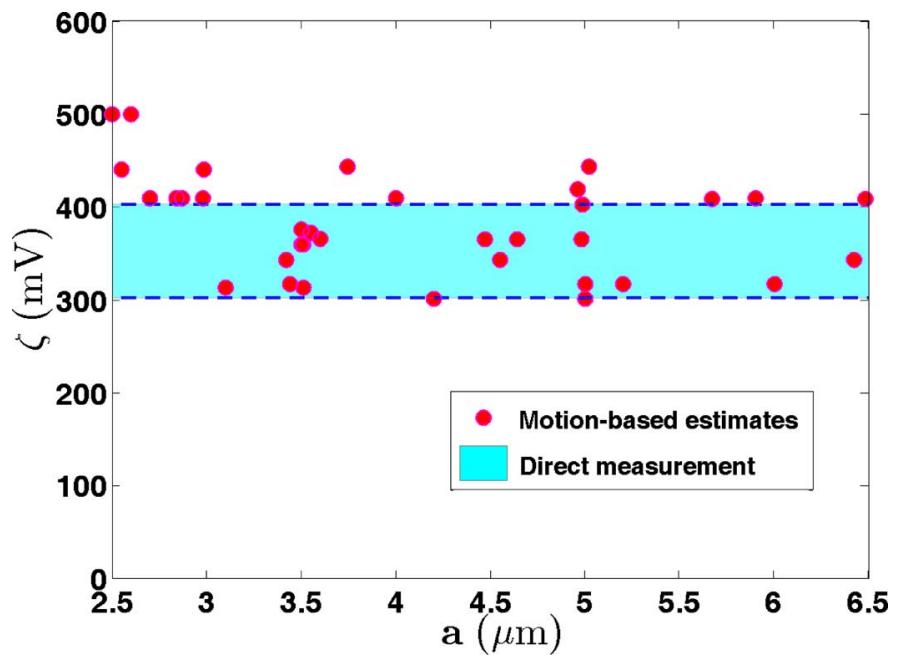

Fig. 7. Comparison of estimated and measured values of zeta-potential among a total of 35 silicon nanowires.

steer the nanowire (around $8 \mu \mathrm{m}$ long) to follow a given path with an average error around $2-4 \mu \mathrm{m}$.

\section{Motion-Planning Results}

We first use simulation to demonstrate and compare the performance of the various motion-planning algorithms. For an $N \times N$ electrode array, we first select the locations of starting and target points $\mathcal{S}$ and $\mathcal{T}$. Depending on the size $N$, we choose the locations $\mathcal{S}$ and $\mathcal{T}$ randomly such that the distance between them is at least $\sqrt{2} / 2 L$ for $N=2$ and $\sqrt{2}(N-2) L$ for $N=$ $3, \ldots, 8$. To eliminate the performance variation, we conduct ten runs for each algorithm and then compute the statistics of these simulation runs.

Fig. 10(a) shows the comparison results of the computation time, while Fig. 10(b) and (c) show the planned travel time for nanowires and the root mean square errors (RMSE) of the trajectories benchmarked by the BFS algorithm. From the results shown in the figure, the computation time of the BFS algorithm increases exponentially with dimension $N$. The complexity of the $A^{*}$, RRT and MinCost algorithms are similar. The heuristic algorithm AllPower shows the best complexity performance among all algorithms. The traveling time and accuracy confirm a similar observation: RRT shows the worst results and all other algorithms demonstrate similar good performance.
Fig. 11 shows the planning trajectories by various algorithms for a $4 \times 4$ electrode array. For clarity, the starting and target points and search area $\mathcal{A}_{m}$ are marked in the figure. Table I lists the travel time, RMSE and $R^{2}$ comparisons. Again, the RMSE and $R^{2}$ results in the table are benchmarked with the BFS algorithm. From these results, we observe that: 1) $A^{*}$ shows superior accuracy performance compared to the other three algorithms; 2) the heuristic algorithms such as MinCost and AllPower show similar accuracy and traveling-time performance; these two algorithms show superior accuracy compared to RRT; 3) all algorithms demonstrate similar path trajectories, except that the trajectory by RRT shows some significant deviations; and 4) the RRT algorithm takes significantly much more time than any of the other algorithms. In summary, the two heuristic algorithms, particularly AllPower, clearly outperform the other three planning approaches in complexity, and yield comparable travel time and accuracy. The heuristic algorithms are less computationally expensive than the $\mathrm{A}^{*}$ and RRT algorithms since they require little or no searching actions in each time step. Although the heuristic algorithms cannot guarantee optimality, they yield comparable travel time and accuracy performance from both the simulation and experimental results.

Fig. 12 shows the motion-planning experiments. In the figure, a silicon nanowire was driven from points $\mathcal{S}$ to $\mathcal{T}$ across the line connecting two electrode centers. Two motion planning algorithms, BFS and AllPower, are implemented. We chose the AllPower algorithm here because it outperforms the other algorithms in both complexity and travel time as demonstrated previously, while the BFS is used as a benchmark for the optimal trajectory. The BFS-based motion trajectory was obtained offline due to the high computational cost. For the AllPower algorithm, the motion planning and control were conducted online. It is clearly shown in the figure that both algorithms give almost the same trajectory path. The zoom-in subplot in Fig. 12 shows that the AllPower trajectory follows closely to the BFS planned trajectory and both algorithms have demonstrated a zigzag pattern motion due to the switching of powered-"on" electrodes at consecutive time steps.

It is interesting to observe that both the BFS and AllPower algorithms generate a similar trajectory that is not the shortest distance between points $\mathcal{S}$ and $\mathcal{T}$. For comparison, we also implemented a motion planning and control design that follows the straight-line (shortest distance) trajectory between $\mathcal{S}$ and $\mathcal{T}$, as shown in Fig. 12. The travel times from $\mathcal{S}$ to $\mathcal{T}$ are 1180, 993, and $977 \mathrm{~s}$ for the straight-line, AllPower and BFS algorithms, respectively. Clearly, the shortest-distance trajectory does not demonstrate the shortest time. This is because that the nanowire motion is determined by the electric-field direction and magnitude. The velocity magnitude is larger along the BFS and AllPower trajectories than along the straight-line trajectories. In particular, since the strength of electric field is inversely proportional to the square of the distance between the particle and electrodes, the nearby electrodes have a more significant impact on the nanowire motion than far-away electrodes. As a result, in the AllPower and BFS trajectories, the nanowire moves rapidly in the horizontal direction when the nearby electrodes are powered on, and then more slowly (but for a shorter distance) when they are turned off. Overall, the velocity of the AllPower tra- 


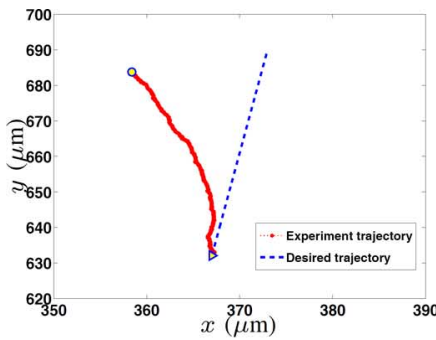

(a)

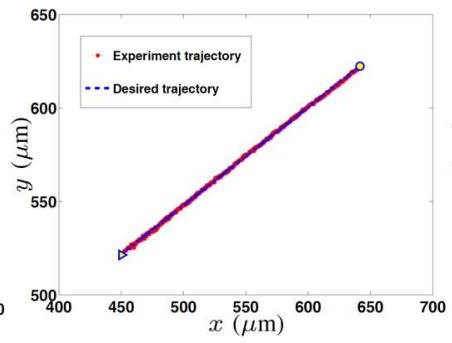

(b)

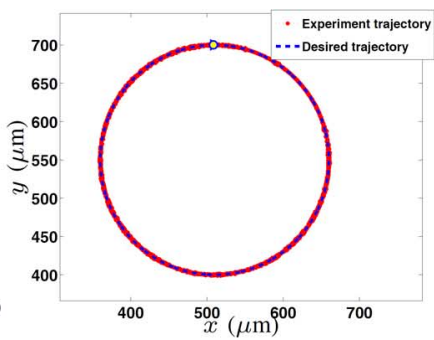

(c)

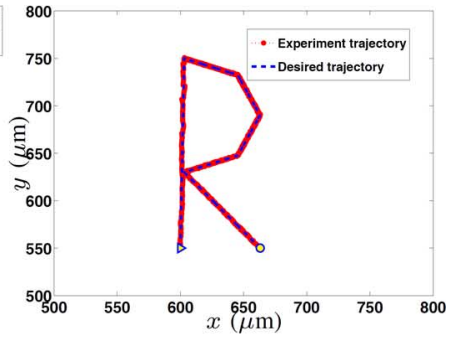

(d)

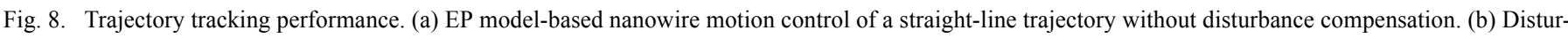

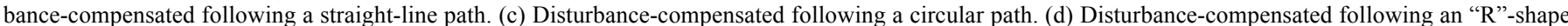
path. In all plots, the triangular and empty circular marks indicate the starting and ending points, respectively, of the nanowire trajectory.

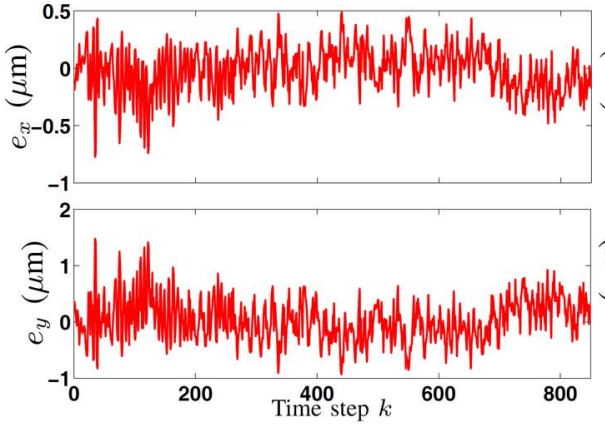

(a)

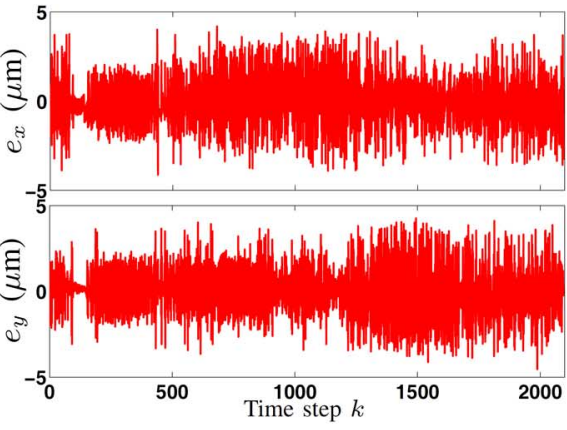

(b)

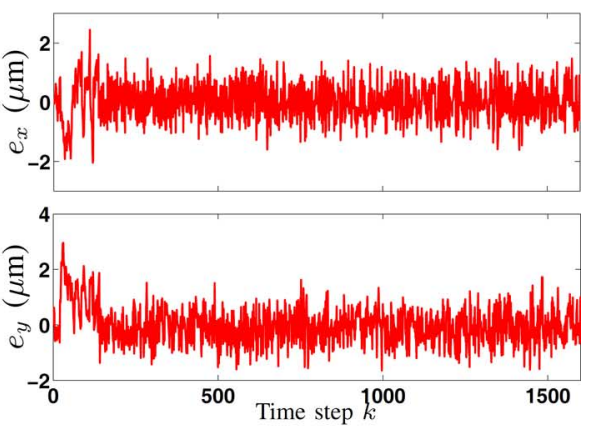

(c)

Fig. 9. Trajectory-tracking errors. (a) Errors for a straight-line trajectory. (b) Errors for a circular trajectory. (c) Errors for an "R"-shape trajectory.

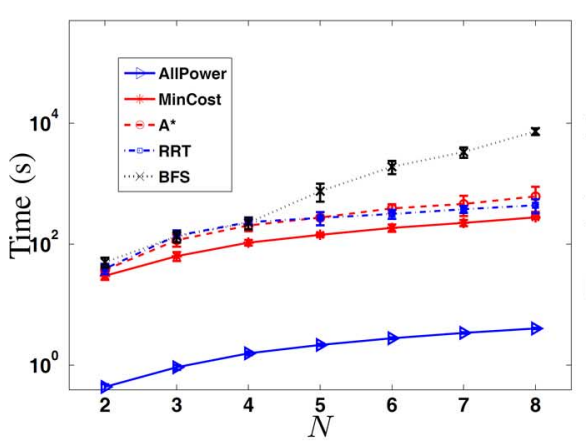

(a)

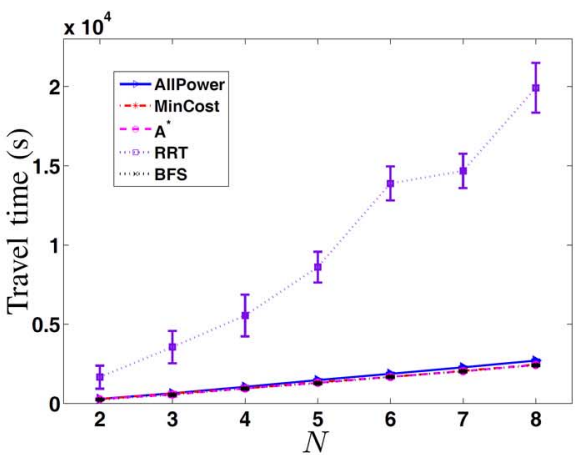

(b)

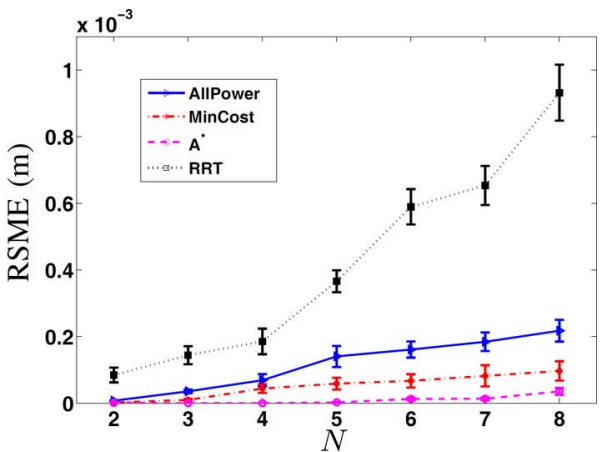

(c)

Fig. 10. Performance comparison among various motion planning algorithms on an $N \times N$ electrode array. (a) Computation time (mean and standard derivation). (b) Nanowire travel time. (c) RMSE (benchmarked with the BFS results).

jectory is significantly larger than that of the straight-line trajectory. Therefore, the fastest trajectory is not always along the shortest distance between two points.

\section{Steering and Manipulating Nanowires}

In this section, we demonstrate the use of the motion planning and control design for 3D nanowire manipulation. We first demonstrate how to steer, orient, and deposit a single nanowire on the device substrate and then present the sequential manipulation and deposition of multiple nanowires.

Fig. 13 illustrates a sequence of snapshots of steering, orientation and manipulation of a single nanowire from location $A$ in fluid suspension to location $B$ on device substrate surface. First, the nanowire was steered from $A$ to $B$ by the AllPower and motion control algorithms [Fig. 13(a) and (b)]. Then, by turning on the ITO electrode on the top plate, an electric field along the vertical $\left(E_{z}\right.$ axis) direction was generated such that the nanowire was aligned vertically and moving towards the bottom surface [Fig. 13(c)]. Finally, once the nanowire reached the surface, the ITO electrode was turned off and the electrodes on the bottom surface were turned on again to reorientate and deposit the nanowire on the substrate in the desired direction, as shown in Fig. 13(d). Due to change in the focal plane of the microscope for the images, several other nanowires and objects on the bottom surface that are seen clearly in Fig. 13(d) are not seen in the other images. Fig. 13(e) shows the overall trajectory of the nanowire by overlaying the image sequence that is shown in part in Fig. 13(a)-(d). 


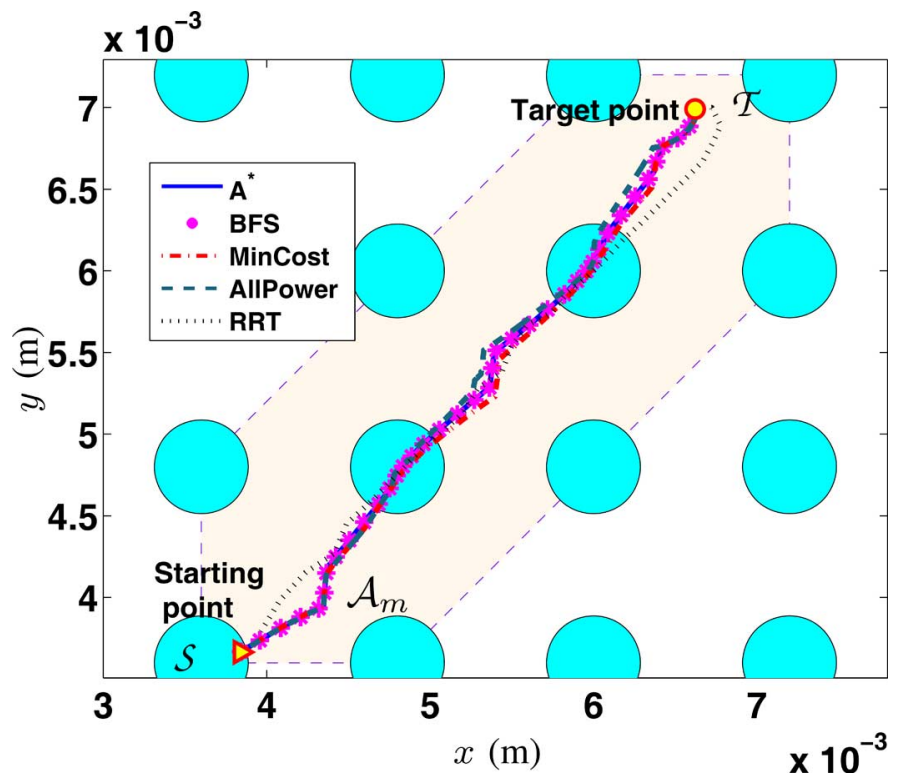

Fig. 11. Comparison of the nanowire trajectories under different motion planning algorithms using a $4 \times 4$ electrode array.

TABLE I

Performance Comparisons for the EXAmple SHOWn IN Fig. 11.

\begin{tabular}{|c|c|c|c|c|c|}
\hline & BFS & AllPower & MinCost & $A^{*}$ & RRT \\
\hline Travel time (s) & 947 & 1062 & 1046 & 950 & 6875 \\
\hline RMSE & N/A & $6.9 \times 10^{-5}$ & $5.9 \times 10^{-5}$ & 0 & $1.4 \times 10^{-4}$ \\
\hline$R^{2}$ & N/A & 0.995 & 0.996 & 1 & 0.978 \\
\hline
\end{tabular}

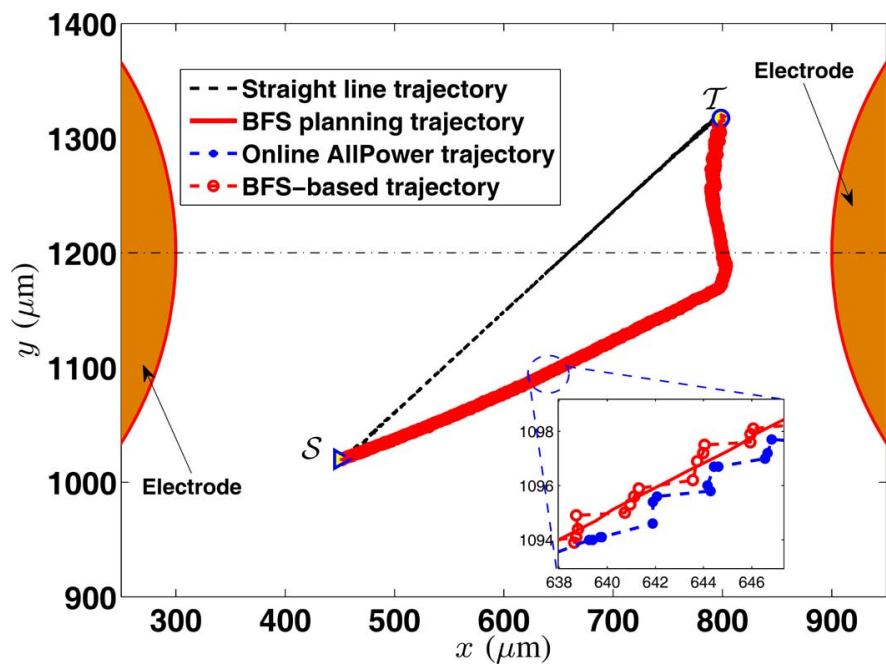

Fig. 12. Experimental silicon-nanowire trajectories under BFS and AllPower Note that the straight-line trajectory shown for comparison is not the minimumtime trajectory.

Fig. 14 further demonstrates the use of the EP-based motion control and manipulation to sequentially steer and deposit three individual nanowires to form larger patterns on the device substrate surface. In Fig. 14(a), three nanowires were suspended in the fluid in an area of a size of $50 \times 100 \mu \mathrm{m}^{2}$. Then, each of these three nanowires was sequentially steered and transferred to the substrate, as shown in Fig. 14(b), using the procedure discussed above. Once the nanowire has settled down on the bottom substrate, it sticks to the surface due to Van der Waals interactions. The nanowire position and orientation is no longer changed by the electric field when moving and reorientating the other nanowires. Thus, we can sequentially deposit nanowires head-to-tail to form a longer, straight-line pattern on the substrate surface.

Fig. 15 demonstrates a few more examples of the use of electric-field-based forces to drive and steer multiple nanowires to form more complicated geometric shapes than those in Fig. 14. Fig. 15(a) shows the result of moving and depositing six nanowires in a straight line, and Fig. 15(b) shows the formation of a hexagonal pattern with multiple nanowires, while Fig. 15(c) demonstrates cross patterns formed by depositing three nanowires perpendicularly on top of three other aligned nanowires. These experiments further confirm the feasibility of using electric-field-based manipulation for fabricating functional nanodevices using nanowires and nanotubes.

\section{CONCLUSION AND FUTURE WORK}

We have presented electric-field-based motion control, planning and manipulation of nanowires in liquid suspension. The motion-planning-and-control schemes take advantage of the symmetry of the electric field generated by the electrodes. A superposition approach was developed to efficiently compute the electric-field distribution for any given set of powered-"on" electrodes. The EP driving force on the particles is modeled, and the vision-based feedback control compensates for unmodeled particle dynamics such as disturbances due to EO flow. The nanowire motion-planning problem is NP hard and we proposed two heuristic algorithms based on the reduced search areas. We compared the heuristic algorithms with other existing motion planning algorithms such as RRT and $A^{*}$. The results showed that the heuristic algorithms significantly reduced the computational complexity while maintained comparable performance with those of the RRT, $A^{*}$ and other algorithms. Extensive experimental results confirmed the analysis and the design of nanowire motion control, planning, and manipulation. Using an additional top electrode, we have also demonstrated the ability to position individual nanowires vertically as well as horizontally and then deposit them in sequence at desired locations on the device substrate.

We are currently designing and fabricating multilayer micro-fluidic devices to test the large-scale motion planning algorithms. Developing more systematic control methods to achieve optimal performance is also among ongoing research tasks. Finally, we are developing the planning-and-control algorithms for simultaneously manipulating multiple nanowires in 3D configurations; this is a critical step toward scalable nano-manufacturing with nanowires and nanotubes.

\section{APPENDIX A}

ProOF OF LEMMA 1

The proof of Lemma 1 extends the approach in [23] and we here discuss the continuous-time case. 


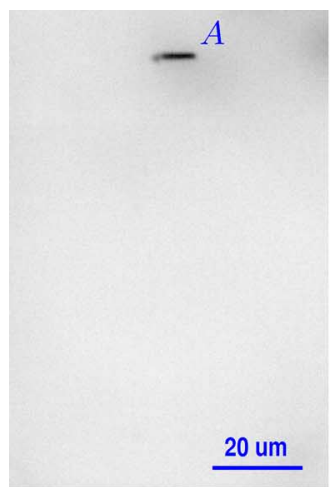

(a)

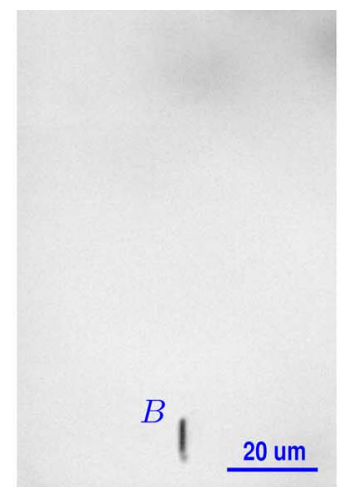

(b)

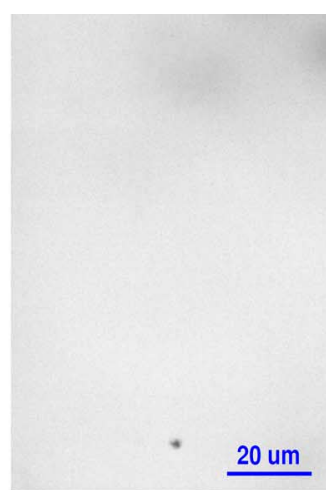

(c)

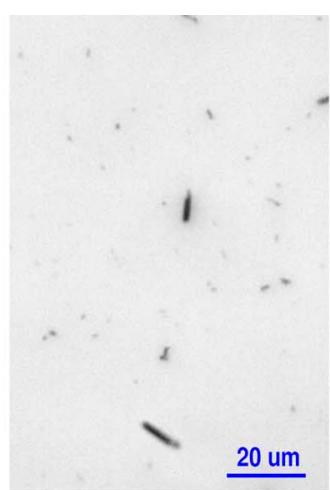

(d)

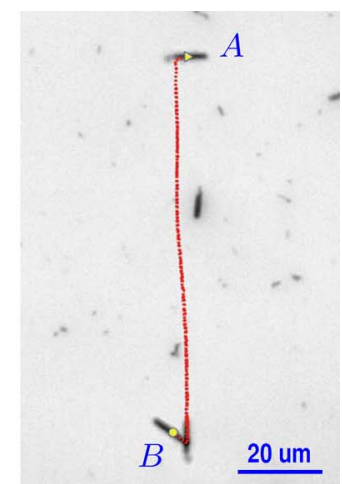

(e)

Fig. 13. Vertical positioning and deposition of a single nanowire on the device substrate. (a) Nanowire at initial location $A$. (b) Nanowire is steered to location $B$ by AllPower algorithm. (c) Using the top ITO electrode, the nanowire was aligned along the vertical direction and steered to touch the bottom surface. (d) By turning the electrode again, the nanowire was reorientated and deposited on the substrate surface with the desired orientation. (e) Overlay trajectory of the entire nanowire motion. The triangular and empty circular marks indicate the starting and ending points of the nanowire.

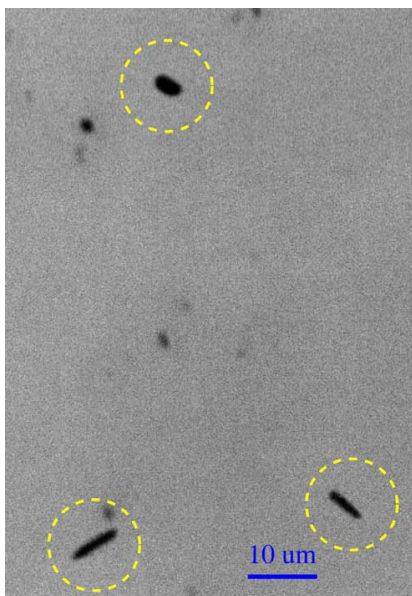

(a)

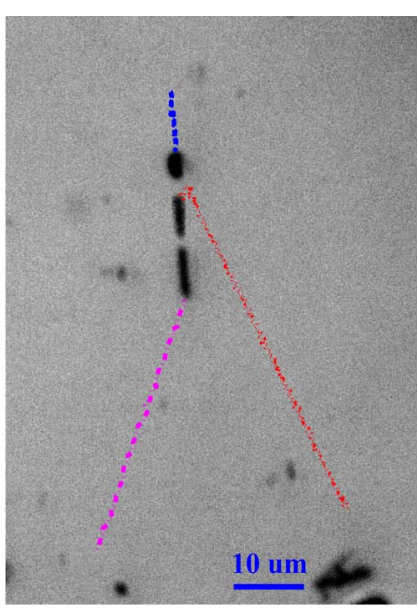

(b)
Fig. 14. Electric-field-based steering and manipulation of three nanowires to form a straight-line on the device substrate. (a) Initial positions of the three nanowires. (b) Final positions of these three nanowires after repositioning and depositing to align into a straight-line.

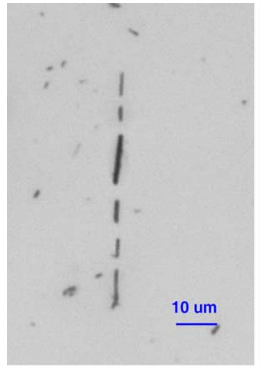

(a)

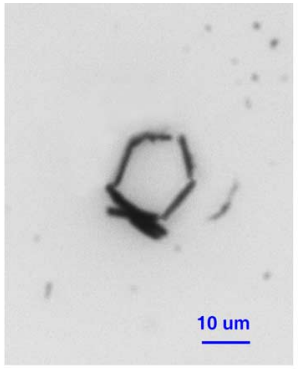

(b)

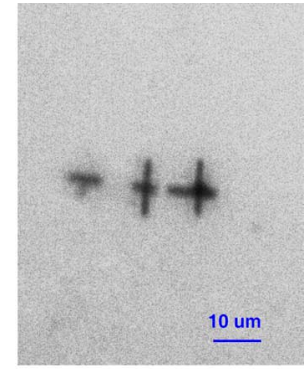

(c)
Fig. 15. Electric-field-based steering and manipulation of multiple nanowires to form: (a) a Straightline, (b) Hexagonal, and (c) Three cross-shape patterns on the device substrate.

Let us denote the nanowire 2D position vector $\boldsymbol{q}=[x y]^{T}$ and error (9) is then written as $\boldsymbol{e}(\boldsymbol{q}, \tau)=\left[e_{x} e_{y}\right]^{T}$. We denote
$\boldsymbol{\Delta}=\left[\begin{array}{ll}\Delta_{x} & \Delta_{y}\end{array}\right]^{T}=\left[\begin{array}{ll}2 \theta_{x} & 2 \theta_{y}\end{array}\right]^{T}$. The potential function (8) is written as

$$
U(\boldsymbol{\Delta})=\frac{1}{2} \beta_{1}\left[\left(1-\cos \Delta_{x}\right)+\left(1-\cos \Delta_{y}\right)\right] .
$$

Note that $U(\boldsymbol{\Delta})$ is lower bounded, i.e., $U(\boldsymbol{\Delta}) \geqslant 0$, and the time derivative of $U(\boldsymbol{\Delta})$ is

$$
\frac{d(U(\boldsymbol{\Delta}))}{d t}=\frac{1}{2} \beta_{1} \sum_{i=x, y} \sin \left(\Delta_{i}\right) \dot{\Delta}_{i}=\beta_{1} \sum_{i=x, y} \frac{\sin \Delta_{i}}{1+e_{i}^{2}} \dot{e}_{i}
$$

where we use the fact $\dot{\Delta}_{i}=2 \dot{\theta}_{i}=2 /\left(1+e_{i}^{2}\right) \dot{e}_{i}, i=x, y$.

Considering $\dot{\boldsymbol{e}}=(\partial \boldsymbol{e} / \partial \boldsymbol{q}) \dot{\boldsymbol{q}}$ and if $\dot{\boldsymbol{q}}=\boldsymbol{v}^{d}$ given by (10), we have

$$
\dot{\boldsymbol{e}}=\left[\begin{array}{ll}
\frac{\partial e_{x}}{\partial x_{x}} & \frac{\partial e_{x}}{\partial y} \\
\frac{\partial e_{y}}{\partial x} & \frac{\partial e_{y}}{\partial y}
\end{array}\right] \boldsymbol{v}^{d}=-\lambda_{2}\left[\begin{array}{c}
\sin \Delta_{x} \\
\sin \Delta_{y}
\end{array}\right]-\lambda_{3}\left[\begin{array}{c}
\hat{e}_{x} \\
\hat{e}_{y}
\end{array}\right] .
$$

Using error prediction (11), i.e., $\hat{\boldsymbol{e}}=\alpha_{1} \boldsymbol{e}+\alpha_{2} \dot{\boldsymbol{e}},(16)$ is written as

$$
\dot{e}=-\lambda_{2}\left[\begin{array}{c}
\sin \Delta_{x} \\
\sin \Delta_{y}
\end{array}\right]-\lambda_{3}\left(\alpha_{1} \boldsymbol{e}+\alpha_{2} \dot{\boldsymbol{e}}\right)
$$

and thus, we obtain

$$
\dot{\boldsymbol{e}}=-\frac{1}{1+\alpha_{2} \lambda_{3}}\left(\lambda_{2}\left[\begin{array}{c}
\sin \Delta_{x} \\
\sin \Delta_{y}
\end{array}\right]+\alpha_{1} \lambda_{3} \boldsymbol{e}\right) .
$$

Plugging (17) into (15), we obtain

$$
\frac{d(U(\boldsymbol{\Delta}))}{d t}=\frac{-\beta_{1}}{1+\alpha_{2} \lambda_{3}} \sum_{i=x, y}\left(\frac{\lambda_{2} \sin ^{2} \Delta_{i}}{1+e_{i}^{2}}+\frac{\alpha_{1} \lambda_{3} e_{i} \sin \Delta_{i}}{1+e_{i}^{2}}\right) .
$$

Noticing that $e_{i} \sin \Delta_{i}=\tan \theta_{i} \sin 2 \theta_{i}=2 \sin ^{2} \theta_{i}$ for $i=x, y$, the above equation becomes

$$
\frac{d(U(\boldsymbol{\Delta}))}{d t}=\frac{-\beta_{1}}{1+\alpha_{2} \lambda_{3}} \sum_{i=x, y} \frac{\lambda_{2} \sin ^{2} \Delta_{i}+2 \alpha_{1} \lambda_{3} \sin ^{2} \theta_{i}}{1+e_{i}^{2}} \leqslant 0
$$

for uniformly positive $\lambda_{2}>0$ and positive $\lambda_{3}, \beta_{1}, \alpha_{1}, \alpha_{2}$. The result (18) implies that $U(\boldsymbol{\Delta})$ converges and thus $\boldsymbol{\Delta}$ converges to a critical point of $U(\boldsymbol{\Delta})$, i.e., $\boldsymbol{\theta}=\mathbf{0}$. Since $\boldsymbol{e}=\tan \boldsymbol{\theta}$, we have $x \rightarrow x_{d}$ and $y \rightarrow y_{d}$ and this completes the proof. 


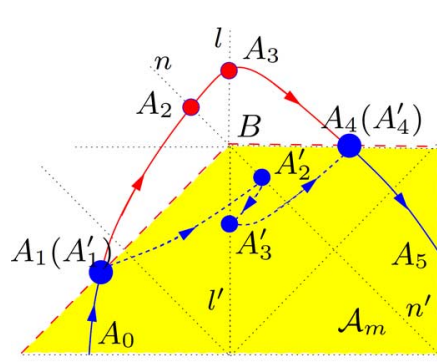

(a)

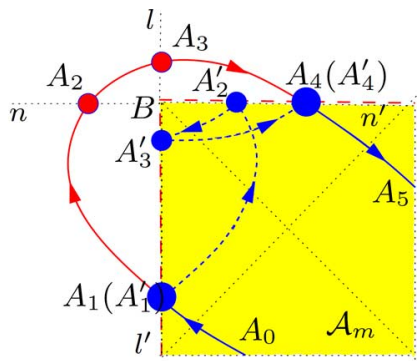

(b)

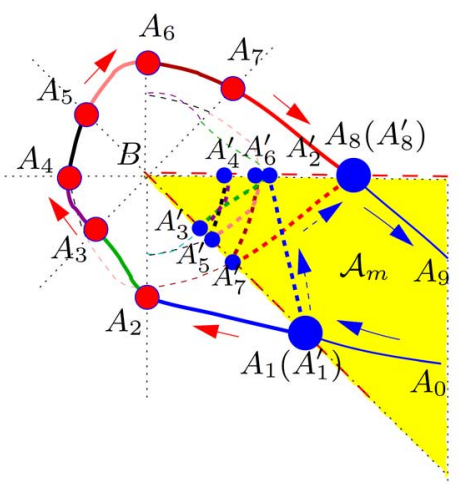

(c)

Fig. 16. Schematic of time-equivalent trajectory within $\mathcal{A}_{m}$. (a) Vertex $B$ with $135^{\circ}$ inner angle. (b) Vertex $B$ with $90^{\circ}$ inner angle. (c) Vertex $B$ with $45^{\circ}$ inner angle.

\section{APPENDIX B}

\section{PROOF OF LEMMA 2}

As shown in Fig. 4, suppose that the minimum-time trajectory $\overparen{A_{1} M A_{2}}$ passes across the mirror line $\overparen{A_{1} A_{2}}$ twice. By Property 1 , it is straightforward to find that an alternative trajectory $\overparen{A_{1} M^{\prime} A_{2}}$, symmetric with $\overparen{A_{1} M A_{2}}$ with respect to mirror line $\overparen{A_{1} A_{2}}$, gives the same time as $\overparen{A_{1} M A_{2}}$. Therefore, the minimum time trajectory can be instead chosen as $\overparen{A_{1} M^{\prime} A_{2}}$ to avoid across line $\overparen{A_{1} A_{2}}$ twice. This completes the proof.

\section{APPENDIX C \\ PROOF OF LEMMA 3}

Due to the page limit, we only present the sketch of the proof here. Since $\mathcal{A}_{m}$ is convex and its boundaries are formed by the mirror lines, each vertex point, say $B$, of $\mathcal{A}_{m}$ has only three possible inner angles: $135^{\circ}, 90^{\circ}$, or $45^{\circ}$, as shown in Fig. 16(a)-(c), respectively. Suppose that a portion of the optimal trajectory is outside $\mathcal{A}_{m}$. We consider the above-mentioned three cases.

For the first two cases, as shown in Fig. 16(a) and (b), let $\overparen{A_{1} \cdots A_{4}}$ denote the minimum time trajectory outside $\mathcal{A}_{m}$. Since boundaries $\overparen{A_{1} B A_{4}}$ of $\mathcal{A}_{m}$ are the mirror lines, $\overparen{A_{1} \cdots A_{4}}$ intersects two other mirror lines $n n^{\prime}$ and $l l^{\prime}$ at $A_{2}$ and $A_{3}$, respectively. By Lemma 2, there exist alternative minimum-time trajectories $\overparen{A_{1}^{\prime} A_{2}^{\prime}}$ and $\overparen{A_{4}^{\prime} A_{3}^{\prime}}$ that are symmetric with $\overparen{A_{1} A_{2}}$ and $\overparen{A_{3} A_{4}}$ about $A_{1} B$ and $A_{4} B$, respectively. Without loss of generality, we assume that both $\overbrace{A_{1}^{\prime} A_{2}^{\prime}}$ and $\overparen{A_{4}^{\prime} A_{3}^{\prime}}$ are located inside $\mathcal{A}_{m}$ because the symmetry lines are the boundaries of
$\mathcal{A}_{m}$ (if only partials of $\overparen{A_{1}^{\prime} A_{2}^{\prime}}$ and $\overparen{A_{4}^{\prime} A_{3}^{\prime}}$ are in $\mathcal{A}_{m}$, applying the similar symmetry multiple times will lead to completely inside $\mathcal{A}_{m}$ as explained in third case). Moreover, there also exists an alternative trajectory $\overparen{A_{2}^{\prime} A_{3}^{\prime}}$ in $\mathcal{A}_{m}$ that gives the same travel time as the rest portion $\overbrace{A_{2} A_{3}}$ of $\overparen{A_{1} \cdots A_{4}}$. Therefore, we find an alternative minimum-time trajectory in $\mathcal{A}_{m}$ that achieves the same time.

For the last case shown in Fig. 16(c), a similar process to the above described can be applied but more complicated procedure is needed to find an alternative minimum-time trajectory inside $\mathcal{A}_{m}$. First, we partition the outside- $\mathcal{A}_{m}$ portion of the minimum-time trajectory $\overparen{A_{1} \cdots A_{8}}$ into seven pieces by their intersections with mirror lines at vertex point $B$; see Fig. 16(c). For each portion $\overparen{A_{i} A_{i+1}}, i=1, \ldots, 7$, a corresponding $\overparen{A_{i}^{\prime} A_{i+1}^{\prime}}$ can be found by mapping symmetrically around the mirror lines multiple times such that $\overparen{A_{i}^{\prime} A_{i+1}^{\prime}}$ generates the same travel time for nanowire motion. Thus, similar to the above cases, the minimum-time trajectory can be instead chosen as $A_{1}^{\prime} \cdots A_{8}^{\prime}$ inside $\mathcal{A}_{m}$. This completes the proof.

\section{ACKNOWLEDGMENT}

The authors thank J. Zheng and Prof. J. Zahn with the Department of Biomedical Engineering, and P. Wang, Y. Zhang, C. Akin, and G. Giraldo with the Department of Mechanical and Aerospace Engineering, Rutgers University, and X. Lu with Nankai University, China, for many helpful discussions and suggestions.

\section{REFERENCES}

[1] A. G. Rinzler, "Materials processing: Sorting out carbon nanotube electronics," Nat. Nanotech, vol. 1, no. 1, p. 17, 2006.

[2] N. Xi and W. J. Li, "Editorial: Recent development in nanoscale manipulation and assembly," IEEE Trans. Autom. Sci. Eng., vol. 3, no. 3, pp. 194-198, Jul. 2006.

[3] S. J. Koh, "Strategies for controlled placement of nanoscale building blocks," Nanoscale Res. Lett., vol. 2, no. 11, pp. 519-545, 2007.

[4] R. Hunter, Foundations of Colloid Science. Oxford, U.K.: Oxford Univ. Press, 1989.

[5] H. Chen, N. Xi, and G. Li, "CAD-guided automated nanoassembly using atomic force microscopy-based nonrobotics," IEEE Trans. Autom. Sci. Eng., vol. 3, no. 3, pp. 208-217, Jul. 2006.

[6] X. Ye, Y. Zhang, C. Ru, J. Luo, S. Xie, and Y. Sun, "Automated pickplace of silicon nanowires," IEEE Trans. Autom. Sci. Eng., vol. 10, no. 3 , pp. 554-561, Jul. 2013.

[7] P. R. C. Gascoyne and J. V. Vykoukal, "Dielectrophoresis-based sample handling in general-purpose programmable diagnostic instruments," Proc. IEEE, vol. 92, no. 1, pp. 22-42, 2004.

[8] D. Fan, Z. Yin, R. Cheong, F. Q. Zhu, R. C. Cammarata, C. L. Chien, and A. Levchenko, "Subcellular resolution delivery of a cytokine through precisely manipulated nanowires," Nat. Nanotech, vol. 5, pp. $545-551,2010$.

[9] B. Edwards, N. Engheta, and S. Evoy, "Electric tweezers: Experimental study of positive dielectrophoresis-based positioning and orientation of a nanorod," J. Appl. Phys., vol. 102, p. 024913, 2007.

[10] S. Kumar, Z. Peng, H. Shin, Z. L. Wang, and P. J. Hesketh, "AC dielectrophoresis of tin oxide nanobelts suspended in ethanol: Manipulation and visualization," Anal. Chem., vol. 82, pp. 2204-2212, 2010.

[11] R. Probst, Z. Cummins, C. Ropp, E. Eaks, and B. Shapiro, "Flow control of small objects on chip: Manipulating live cells, quantum dots, and nanowires," IEEE Control Syst. Mag., vol. 32, no. 2, pp. 26-53, Apr. 2012.

[12] S. LaValle, Planning Algorithms. Cambridge, U.K.: Cambridge Univ. Press, 2006

[13] E. J. Griffith, S. Akella, and M. K. Goldberg, "Performance characterization of a reconfigurable planar-array digital microfluidic system,' IEEE Trans. Comput.-Aided Design Integr. Circuits Syst., vol. 25, no. 2, pp. 345-357, Feb. 2006. 
[14] P.-H. Yuh, C.-L. Yang, and Y.-W. Chang, "Bioroute: A network-flow-based routing algorithm for the synthesis of digitalmicrofluidic biochips," IEEE Trans. Comput.-Aided Design Integr. Circuits Syst., vol. 27, no. 11, pp. 1928-1941, Nov. 2008.

[15] M. Peasgood, C. M. Clark, and J. McPhee, "A complete and scalable strategy for coordinating multiple robots within roadmaps," IEEE Trans. Robot., vol. 24, no. 2, pp. 283-292, Apr. 2008.

[16] A. A. Furtuna, "Minimum time kinematic trajectories for self-propelled rigid bodies in the unobstructed plane," Ph.D. dissertation, Dept. Comput. Sci., Dartmouth College, Hanover, NH, USA, 2011.

[17] K. Yu, X. Lu, J. Yi, and J. Shan, "Electrophoresis-based motion planning and control of nanowires in suspended fluids," in Proc. IEEE Conf. Autom. Sci. Eng., Madison, WI, USA, 2013, pp. 831-836.

[18] K. Yu, J. Yi, and J. Shan, "Motion control and manipulation of nanowires under electric-fields in fluid suspension," in Proc. IEEE/ASME Int. Conf. Adv. Intell., Besancon, France, 2014.

[19] J. Happel and H. Brenner, Low Reynolds Number Hydrodynamics. Hague, The Netherlands: Martinus Nijhoff, 1983.

[20] F. M. Zimmermann and J. W. Shan, "Rotational friction constant of single-wall carbon nanotubes in liquid suspension," Appl. Phys. Lett., vol. 94, p. 053107, 2009.

[21] R. G. Larson, The Structure and Rheology of Complex Fluids. Oxford, U.K.: Oxford Univ. Press, 1999.

[22] Y. Solomentsev and J. L. Anderson, "Electrophoretic transport of spheroidal colloids in nonhomogeneous electric fields," Ind. Eng. Chem. Res., vol. 34, no. 10, pp. 3231-3238, 1995.

[23] P. Y. Li and R. Horowitz, "Passive velocity field control (PVFC): Part II-Application to contour following," IEEE Trans. Autom. Control, vol. 46, no. 9, pp. 1360-1371, Sep. 2001

[24] Y. Zhang and J. Yi, "Velocity field-based maneuver regulation of autonomous motorcycles," in Proc. IFAC Symp. Mechatronic Syst., Cambridge, MA, USA, 2010, pp. 385-392.

[25] K. Peng, J. Hu, Y. Yan, Y. Wu, H. Fang, Y. Xu, S. Lee, and J. Zhu, "Fabrication of single-crystalline silicon nanowires by scratching a silicon surface with catalytic metal particles," Adv. Funct. Mater., vol. 16, no. 3, pp. 387-394, 2006.

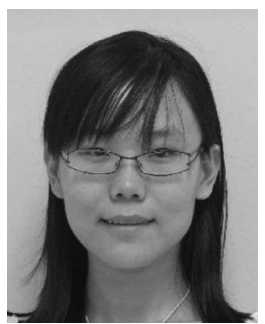

Kaiyan Yu (S'13) received the B.S. degree in intelligent science and technology from Nankai University, Tianjin, China, in 2010. She is currently working towards the Ph.D. degree in mechanical and aerospace engineering at Rutgers University, Piscataway, NJ, USA.

Her current research interests include autonomous robotic systems and control, mechatronics, automation science and engineering, with applications to biomedical systems.

$\mathrm{Ms}$. Yu is a student member of American Society of Mechanical Engineers (ASME)

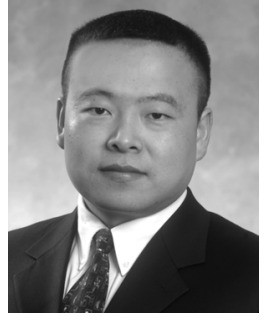

Jingang Yi (S'99-M'02-SM'07) received the B.S. degree in electrical engineering from Zhejiang University, Hangzhou, China, in 1993, the M.Eng. degree in precision instruments from Tsinghua University, Beijing, China, in 1996, and the M.A. degree in mathematics and the Ph.D. degree in mechanical engineering from the University of California, Berkeley, CA, USA, in 2001 and 2002, respectively.

He is currently an Associate Professor of Mechanical Engineering at Rutgers University. His research interests include autonomous robotic systems, dynamic systems and control, mechatronics, automation science and engineering, with applications to biomedical systems, civil infrastructure and transportation systems.

Dr. Yi is a Member of the American Society of Mechanical Engineers (ASME). He is a recipient of the 2010 U.S. National Science Foundation (NSF) CAREER Award. He has coauthored papers that have been awarded several best papers at the IEEE/ASME AIM, ASME DSCC, IEEE ICRA, etc. Dr. Yi currently serves as an Associate Editor for the IEEE TRANSACTIONS on Automation SCIENCE AND ENGINEering, IFAC Journal Control Engineering Practice, and the IEEE Robotics and Automation Society Conference Editorial Board (since 2008). He also served as a Guest Editor for the IEEE TRANSACTIONS ON AUTOMATION SCIENCE AND ENGINEERING in 2009 and an Associate Editor for the ASME Dynamic Systems and Control Division Conference Editorial Board from 2008 to 2010

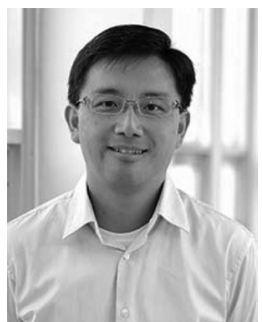

Jerry W. Shan received the B.S. in engineering and applied science, the M.S. in aeronautics, and Ph.D. degree in aeronautics with a minor in electrical engineering in 1995, 1996, and 2001, respectively, all from the California Institute of Technology, Pasadena, CA, USA.

Dr. Shan joined the Mechanical and Aerospace Engineering Department at Rutgers University in 2003, where he is currently an Associate Professor and Department Laboratory Director. His research interests are in fluid dynamics, heat transfer, and nanotechnology. He has experience in experiments and analysis of fluid flows at scales ranging from individual nanoparticles, to vesicles and cells, to large-Reynoldsnumber turbulent flows. His recent work focuses on the mechanics and thermophysical properties of nanoparticle composites and suspensions, with influence from external electromagnetic fields.

Dr. Shan is a 2007 recipient of the National Science Foundation (NSF) CAREER Award, and serves on the editorial board of the Proceedings of the Royal Society A. 